\title{
BEHAVIOUR OF PASSAGE TIME FOR A QUEUEING NETWORK MODEL WITH FEEDBACK: A SIMULATION STUDY
}

\author{
BIDYUT K. MEDYA
}

Received 21 June 2002

\begin{abstract}
The study of the queueing system presented in this note was motivated by its possible application to a multiprogramming computer system. The paper considers a closed network with two service centres with feedback. The steady-state behaviour of the passage time, the random times for a job to traverse a portion of the network, is examined through simulation. The model can be applied to a twin-processor multiprogramming computer system.
\end{abstract}

2000 Mathematics Subject Classification: 60K25.

1. Introduction. In this work, we deal with a two-station closed network where, after service completion at one centre, a customer may move either to the other centre or to the tail of the queue at the same centre. Feedback probabilities are specified for each centre. Also for each centre, we assume service times to be independent and exponentially distributed to ensure Markovian character.

This type of models arises in theoretical computer science, more specifically regarding multiprocessor multiprogramming environments.

We can think of a computer system based on $M$ parallel processors which can handle $N$ statistically identical jobs simultaneously. The jobs circulate among the processors completing identical partial processing. Such a system can be called an $M$-processor system with $N$ degree of multiprogramming. The processors are the service nodes and the programs are identified with the jobs. Thus we have a typical situation of closed network with feedback.

The augmented job stack process helps to identify the position of the process at any instant.

We study the behaviour of passage times which are the random times for a job to traverse a portion of the network.

We use the method of regenerative simulation to find the steady-state distribution of passage time and the distribution of the number of transitions constituting a passage time.

Steady-state distribution has been considered in Medya [2] along with supporting simulation studies.

Yechiali [6] and Brandon and Yechiali [1] consider $N$-node open network systems with feedback to the first node from an optimal sequencing point of view. Nguyen [3] considers an interesting situation of mixed network with features of open queue and 


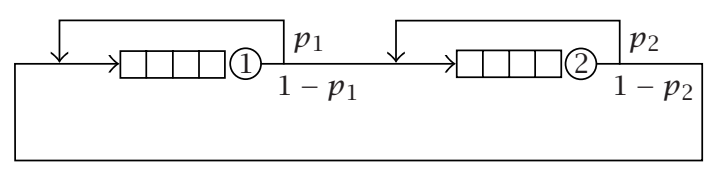

FIGURE 2.1. Two-node closed network model.

closed network with feedback. A model with feedback at one node only [5] can be used for a multiprogramming system with single processor and single I/O channel. This model has been discussed in [4], so this model is a special case of the model which we have considered with the second feedback probability taken as zero.

2. The model. The type of model considered in this work is a queueing system consisting of two single-server service centres and a fixed number, $N$, of jobs (Figure 2.1). After service completion at centre $i, i \in\{1,2\}$, a job moves instantaneously to the tail of the queue at centre $i$ with fixed probability $p_{i}\left(0 \leq p_{i}<1\right)$ and with probability $\left(1-p_{i}\right)$, moves to the tail of the queue at centre $i^{\prime}$, where $i^{\prime}=2,1$, for $i=1,2$, respectively. We assume that both queues are served according to a first-come first-served (FCFS) discipline. We also suppose that all service times are mutually independent and that the service times at centre $i$ are identically distributed as a positive random variable, $L_{i}$, $i=1,2$. In this work we assume $L_{i}$ to have exponential distribution with parameter $\lambda_{i}$.

Let $X(t)$ be the number of jobs waiting or in service at centre 1 at time $t$. The process $\{X(t): t \geq 0\}$ is a generalised semi-Markov process (GSMP) with finite state space, $S=$ $\{0,1,2, \ldots, N\}$, and event set, $E=\left\{e_{1}, e_{2}\right\}$, where event $e_{i}$ can be defined as "service completion at centre $i$." For $s \in S$, the sets $E(s)$ of events that can occur in state $s$ are as follows. The event $e_{1} \in E(s)$ if and only if $0<s \leq N$, and the event $e_{2} \in E(s)$ if and only if $0 \leq s<N$. If $e=e_{2}$, then the state transition probability $p(s-1 ; s, e)=1-p_{1}$ and $p(s ; s, e)=p_{1}$ when $0<s \leq N$; if $e=e_{2}$, then $p(s+1 ; s, e)=1-p_{2}$ and $p(s ; s, e)=p_{2}$ when $0 \leq s<N$. All other state transition probabilities $p\left(s^{\prime} ; s, e\right)$ are equal to zero.

The model is appropriate for a twin-processor multiprogramming computer system where $N$ denotes the degree of multiprogramming and programs can be either transferred from one processor to the other or fed back to the same processor.

\section{Augmented job stack process}

Jов STACK. It is an enumeration by service centre of all the jobs of the network.

In our model the job stack process is called $Z(t)$, where $Z(t)$ is the number of jobs waiting or in service at centre 1 at time $t$. It is an irreducible continuous time Markov chain (CTMC) with finite state space [5, page 67].

The augmented job stack process for this model is

$$
X(t)=\{Z(t), N(t): t \geq 0\},
$$

where $Z(t)$ denotes the number of jobs waiting or in service at centre 1 at time $t$ and $N(t)$ denotes the position from the top (i.e., left in Figure 2.1) of the marked job in the job stack at time $t$. 
The state space, $G^{*}$, is

$$
G^{*}=\{(i, j): i \in\{0,1, \ldots, N\}, j \in\{1,2, \ldots, N\}\} .
$$

PASSAGE TIME. Informally, passage times in a network of queues are the random times for a job to traverse a portion of the network.

Passage times for the marked job are specified by means of four nonempty subsets $\left(A_{1}, A_{2}, B_{1}\right.$, and $\left.B_{2}\right)$ of the state space $G^{*}$, of the augmented job stack process $X$. The sets $A_{1}, A_{2}$ (resp., $B_{1}, B_{2}$ ) jointly define the random times at which passage times for the marked job start (resp., terminate). The sets $A_{1}, A_{2}, B_{1}$, and $B_{2}$ in effect determine when to start and stop the clock measuring a particular passage time of the marked job.

Here we consider the passage time, $P$, that starts when a job completes service at centre 2 (and joins the tail of the queue at centre 1) and terminates at the next such time the job completes service at centre 2 (and joins the tail of the queue at centre 1 ).

The passage time is specified by the sets

$$
\begin{aligned}
& A_{1}=B_{1}=\{(i, N): 0 \leq i<N\}, \\
& A_{2}=B_{2}=\{(i+1,1): 0<i \leq N\} .
\end{aligned}
$$

STEADY-STATE BEHAVIOUR. Under fairly general conditions, which are satisfied here, the passage times have a steady-state distribution, that is, if $T_{n}$ denotes the $n$th passage time, then $T_{n} \Rightarrow T$ for some random variable $T$. The distribution of $T$ will be referred to as the steady-state distribution of passage time.

4. Simulation. To simulate the process, the method of regenerative simulation (Shedler [5]) has been employed here.

REgENERATIVE METHOD FOR SIMULATION ANALYSIS. We have seen that in the presence of certain regularity conditions, a regenerative stochastic process $\{X(t): t \geq$ $0\}$ has a limiting distribution provided that the expected time between regeneration points is finite. Furthermore, the regenerative structure ensures that the behaviour of the regenerative process in a cycle determines the limiting distribution of the process as a ratio of expected values. A consequence of these results is that a strongly consistent point estimate and asymptotic confidence interval for the expected value of the general (measurable) function of the limiting random variable can be obtained by observing a finite portion of a single sample path of the process. This comprises the regenerative method and is accomplished by simulating the process in cycles and measuring quantities determined by the individual cycles.

CrClE. Cycles are defined by intervals between successive returns to state $(1,1)$. 
4.1. Simulation for the distribution of passage time. Here a sample path for the process is generated through simulation through a large number of cycles, where a cycle starts and ends with the state $(1,1)$. Passage times are marked on the sample path. The parameters to be estimated,

$$
P_{n}=P(n \leq T<n+1)=E\left[I_{A_{n}}\right],
$$

where $n=0,1,2, \ldots$ and $A_{n}=\{n \leq T<n+1\}$, together determine the probability distribution of $T$. By the basic result of regenerative simulation (Shedler [5]), which is similar to the result in Medya [2], the strongly consistent estimator of $p_{n}$ is

$$
\frac{g\left(A_{n}\right)}{\sum_{n=0}^{n^{*}} g\left(A_{n}\right)},
$$

where $g\left(A_{n}\right)$ is the number of simulated passage times $T$ with $T \in[n, n+1)$ and $n^{*}$ is a suitably chosen upper limit.

We have considered various cases, namely, number of jobs $=2,5,10$ and the combination of $\left(p_{1}, p_{2}\right)$ values: $p_{1}=p_{2}=0.1 ; p_{1}=p_{2}=0.25 ; p_{1}=p_{2}=0.5 ; p_{1}=p_{2}=0.75$; $p_{1}=p_{2}=0.9 ; p_{1}=0.25, p_{2}=0.75 ;$ and $p_{1}=0.1, p_{2}=0.9$.

Because of the symmetry, the last two cases are, respectively, equivalent to $p_{1}=0.75$, $p_{2}=0.25$ and $p_{1}=0.9, p_{2}=0.1$.

ReSUltS FOR PASSAGE TIME Distribution. Figures 4.1, 4.2, 4.3, 4.4, 4.5, 4.6, and 4.7 represent the simulated steady-state distribution of the passage time in various cases. The summary statistics, namely, means, s.d.'s, logarithmic means $[E(\ln T)]$, and skewness and kurtosis coefficients are presented in Table 4.1. The result shows that for a fixed number of jobs and $p_{1}=p_{2}$, the mean and s.d. of the passage time increase with the common value of $p_{1}$ and $p_{2}$. And for unequal values of $p_{1}$ and $p_{2}$, the greater one dominates.

For fixed values of $p_{1}$ and $p_{2}$, the mean and s.d. of the passage time are nearly proportional to the number of jobs. The same is true for the antilog of the logarithmic mean (i.e., geometric mean). When $p_{1}=p_{2}$, both skewness and kurtosis show very little variation, irrespective of the common value and the number of jobs.

When one of $p_{1}$ or $p_{2}$ is large, skewness and kurtosis decrease as the number of jobs increases. In other cases, skewness and kurtosis increase with the number of jobs except for $p_{1}=p_{2}=0.9, N=10$.

For a fixed number of jobs, when $p_{1}=p_{2}$, skewness and kurtosis increase with the common value of $p_{1}$ and $p_{2}$. When $p_{1} \neq p_{2}$, we generally get larger skewness and kurtosis.

4.2. Simulation for the distribution of the number of transitions constituting a passage time. Each passage time is a sum of $M$ exponential service times (transitions) where $M$ is a random variable. We can use the same technique of simulation to estimate 


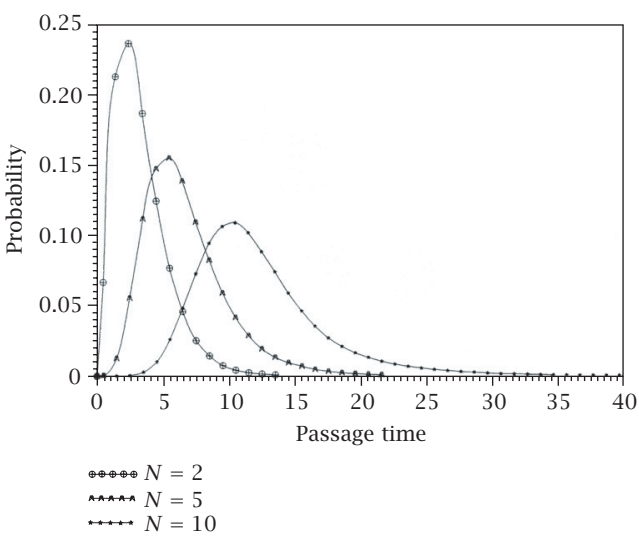

FIGURE 4.1

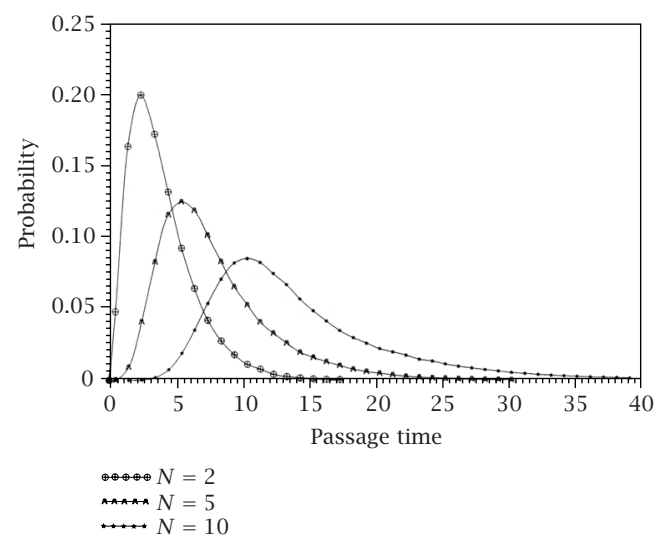

FIGURE 4.2

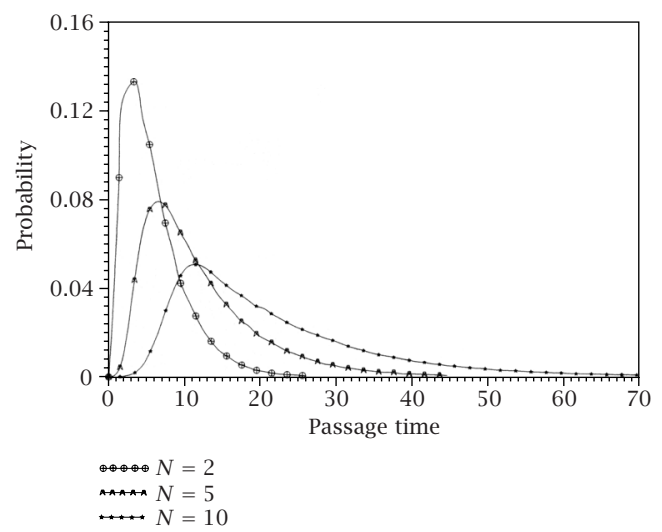

FIGURE 4.3 


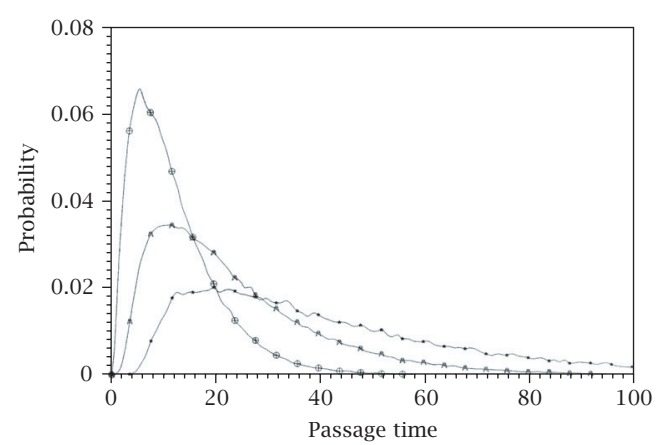

$N=2$

$\rightarrow n+x=5$

แ3 $=10$

FIGURE 4.4

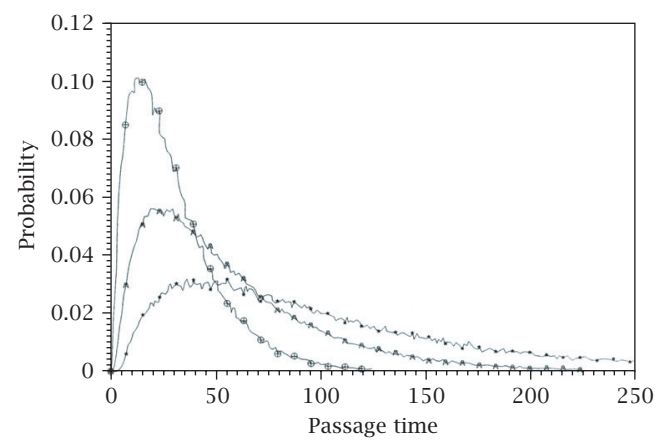

$N=2$

$\rightarrow+\infty=5$

‥ $N=10$

FIGURE 4.5

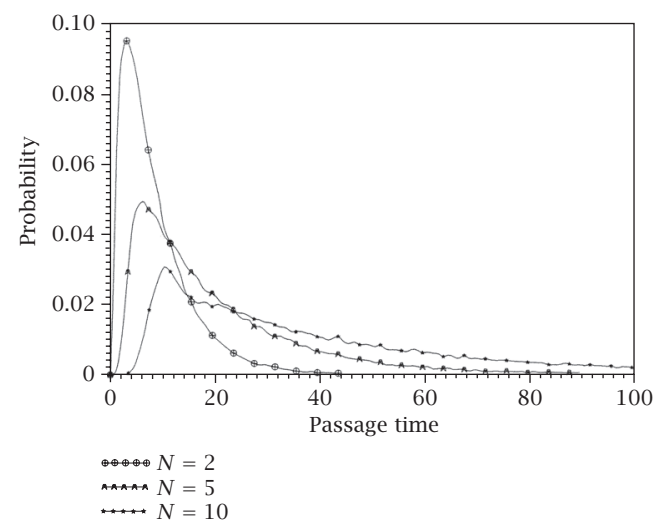

FIGURE 4.6 


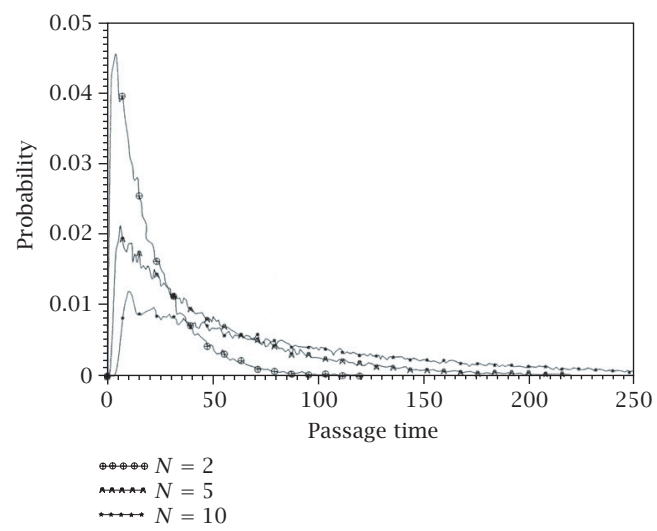

FIGURE 4.7

TABLE 4.1 Summary statistics for distribution of $T$.

\begin{tabular}{l|rrr|rrr}
\hline \multirow{2}{*}{$\left(p_{1}, p_{2}\right)$} & \multicolumn{3}{|c|}{ Mean of $T$} & \multicolumn{3}{c}{ s.d. of $T$} \\
\cline { 2 - 7 } & \multicolumn{1}{|c}{$N=2$} & \multicolumn{1}{|c}{$N=5$} & $N=10$ & \multicolumn{1}{c}{$N=2$} & \multicolumn{1}{c}{$N=5$} & \multicolumn{1}{c}{$N=10$} \\
\hline$(0.1,0.1)$ & 3.33826 & 6.67818 & 12.24155 & 2.02782 & 3.15246 & 4.78688 \\
$(0.25,0.25)$ & 4.02476 & 7.98108 & 14.68056 & 2.57387 & 4.38026 & 7.25242 \\
$(0.5,0.5)$ & 6.03558 & 11.97407 & 22.10263 & 4.08600 & 7.64751 & 13.62768 \\
$(0.75,0.75)$ & 12.01037 & 23.92743 & 43.84632 & 8.44603 & 16.99128 & 31.24527 \\
$(0.9,0.9)$ & 29.98046 & 59.93902 & 99.99991 & 21.40377 & 44.80911 & 79.96751 \\
$(0.25,0.75)$ & 8.68969 & 49.95054 & 39.96846 & 7.07903 & 16.14019 & 33.29985 \\
$(0.1,0.9)$ & 20.04972 & 49.90799 & 97.97700 & 18.59241 & 46.38462 & 87.06717 \\
\hline
\end{tabular}

\begin{tabular}{l|ccc}
\hline \multirow{2}{*}{$\left(p_{1}, p_{2}\right)$} & \multicolumn{3}{|c}{ Logarithmic mean of $T$} \\
\cline { 2 - 4 } & $N=2$ & $N=5$ & $N=10$ \\
\hline$(0.1,0.1)$ & 1.00642 & 1.79213 & 2.43716 \\
$(0.25,0.25)$ & 1.18122 & 1.94203 & 2.58465 \\
$(0.5,0.5)$ & 1.56428 & 2.29804 & 2.93345 \\
$(0.75,0.75)$ & 2.22860 & 2.93506 & 3.54847 \\
$(0.9,0.9)$ & 3.13099 & 3.81310 & 4.32806 \\
$(0.25,0.75)$ & 1.85664 & 2.70072 & 3.38191 \\
$(0.1,0.9)$ & 2.58013 & 3.48973 & 4.17065 \\
\hline
\end{tabular}

\begin{tabular}{l|ccc|rcc}
\hline \multirow{2}{*}{$\left(p_{1}, p_{2}\right)$} & \multicolumn{3}{|c|}{ Skewness of $T$} & \multicolumn{3}{c}{ Kurtosis of $T$} \\
\cline { 2 - 7 } & $N=2$ & $N=5$ & $N=10$ & $N=2$ & $N=5$ & $N=10$ \\
\hline$(0.1,0.1)$ & 1.21470 & 1.30283 & 1.54099 & 5.20290 & 6.04107 & 7.18983 \\
$(0.25,0.25)$ & 1.39587 & 1.61683 & 1.76532 & 6.08617 & 7.27155 & 7.80637 \\
$(0.5,0.5)$ & 1.44394 & 1.64380 & 1.75444 & 5.98575 & 7.21286 & 7.62408 \\
$(0.75,0.75)$ & 1.44972 & 1.60336 & 1.67858 & 6.10992 & 6.77495 & 6.94187 \\
$(0.9,0.9)$ & 1.45591 & 1.58746 & 1.34684 & 6.21520 & 6.62154 & 4.80362 \\
$(0.25,0.75)$ & 2.12381 & 1.88128 & 1.85668 & 11.16495 & 8.04259 & 7.49442 \\
$(0.1,0.9)$ & 2.00422 & 1.95407 & 1.49411 & 9.03806 & 8.39876 & 5.04933 \\
\hline
\end{tabular}




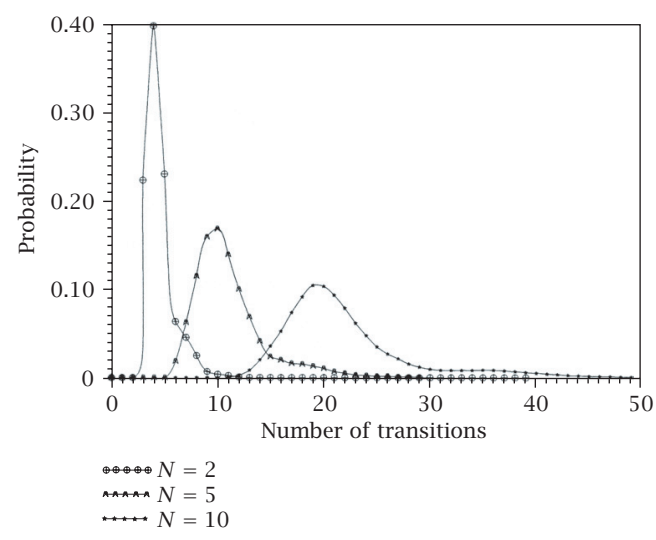

FIGURE 4.8

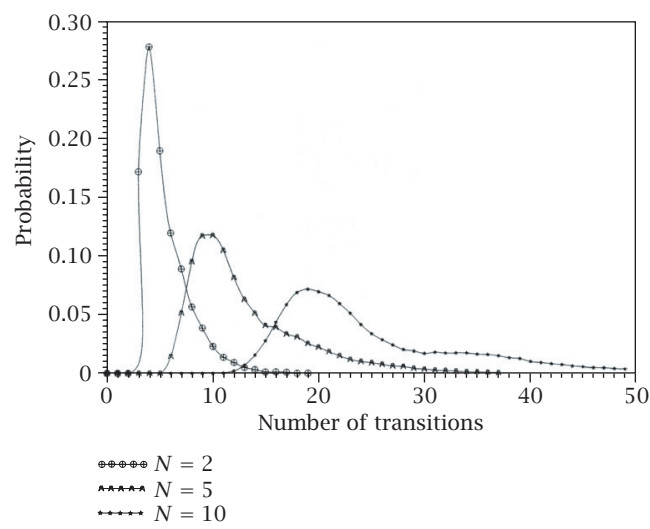

FigURE 4.9

the probability distribution of $M$. The appropriate estimation formula for $Q_{n}=P(M=$ $m)$ is

$$
\widehat{Q_{n}}=\frac{\text { total number of passage times with } m \text { transitions }}{\text { total number of passage times }},
$$

which is derived from the general result for strongly consistent estimators in regenerative simulation. We have considered various cases as mentioned in Section 4.1.

RESULTS FOR THE DISTRIBUTION OF NUMBER OF TRANSITIONS. Figures 4.8, 4.9, $4.10,4.11,4.12,4.13$, and 4.14 represent the simulated steady-state distribution of the number of transitions making up a passage time. We note the oscillations in the distribution for the cases $p_{1}=0.1, p_{2}=0.9$ and $p_{1}=0.25, p_{2}=0.75$, that is, the asymmetric cases (Figures 4.13 and 4.14).

The summary statistics, namely, means, s.d.'s, logarithmic means $[E(\ln M)]$, and skewness and kurtosis coefficients are presented in Table 4.2. 


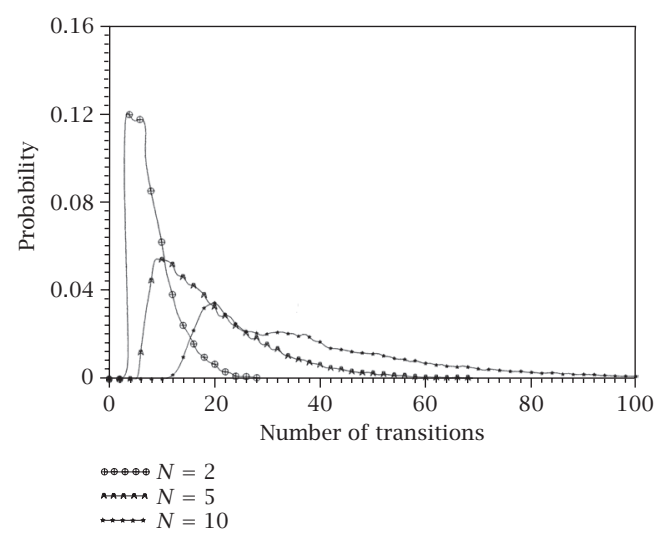

FIGURE 4.10

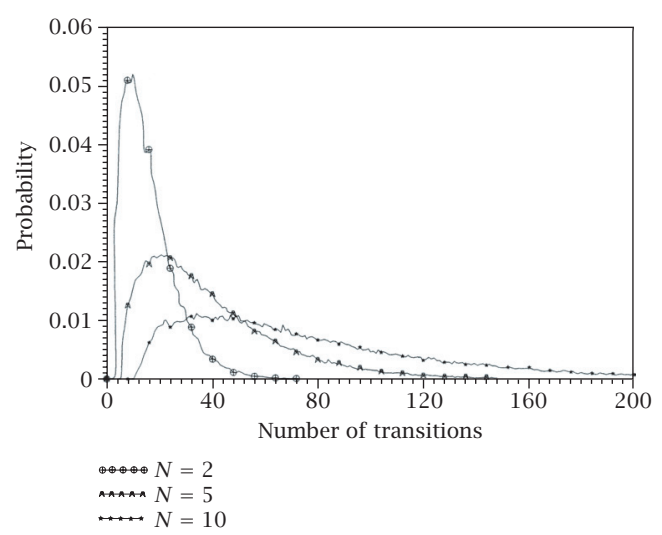

FigURE 4.11

The result shows that for a fixed number of jobs and $p_{1}=p_{2}$, the mean and s.d. of the number of transitions increase with the common value of $p_{1}$ and $p_{2}$. And for unequal values of $p_{1}$ and $p_{2}$, the greater one dominates.

For fixed values of $p_{1}$ and $p_{2}$, the mean and s.d. of the number of transitions are nearly proportional to the number of jobs. The same is true for the antilog of the logarithmic mean (i.e., geometric mean). Both skewness and kurtosis decrease with the increasing number of jobs in cases where $p_{1}$ or $p_{2}$ or both are large. In other cases the variation is small.

For a fixed number of jobs and $p_{1}=p_{2}$, both skewness and kurtosis decrease with the common value of $p_{1}$ and $p_{2}$. Another interesting observation is that skewness and kurtosis show exactly the same pattern. The same is true for the distribution of passage times. 


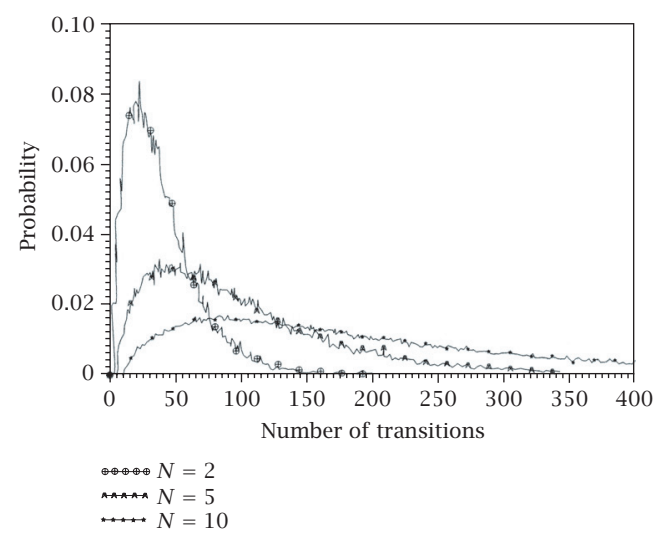

FIGURE 4.12

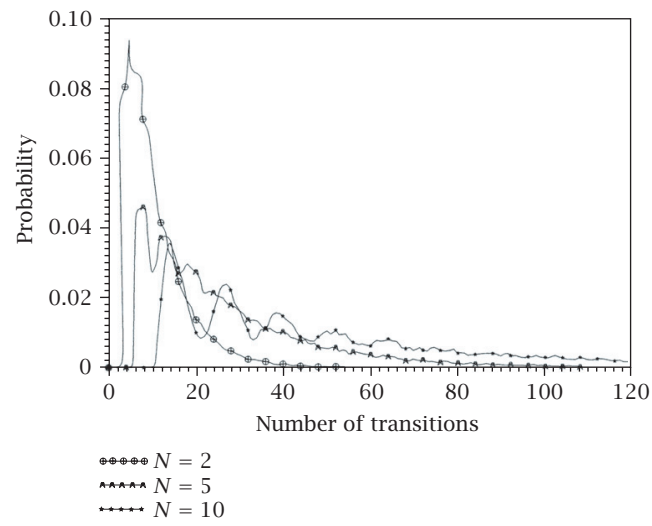

FIGURE 4.13

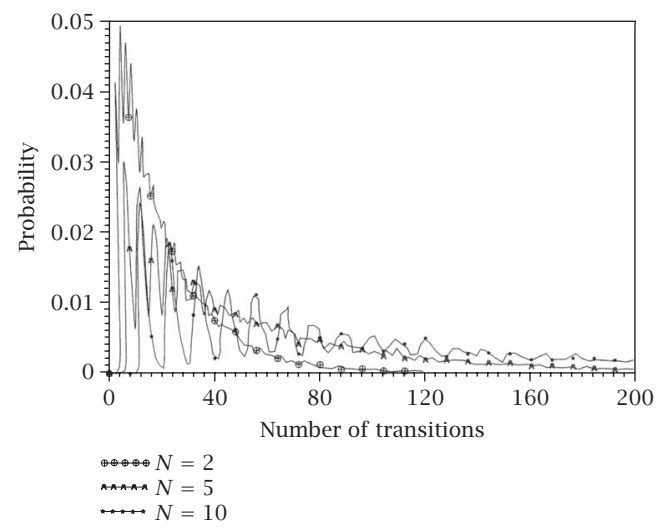

FIGURE 4.14 
TABLE 4.2 Summary statistics for distribution of $M$.

\begin{tabular}{l|rrr|rrr}
\hline \multirow{2}{*}{$\left(p_{1}, p_{2}\right)$} & \multicolumn{3}{|c|}{ Mean of $M$} & \multicolumn{3}{c}{ s.d. of $M$} \\
\cline { 2 - 7 } & \multicolumn{1}{|c}{$N=2$} & $N=5$ & \multicolumn{1}{c}{$N=10$} & \multicolumn{1}{c}{$N=2$} & \multicolumn{1}{c}{$N=5$} & \multicolumn{1}{c}{$N=10$} \\
\hline$(0.1,0.1)$ & 4.45201 & 11.09873 & 22.18072 & 1.34290 & 3.49283 & 6.54112 \\
$(0.25,0.25)$ & 5.38323 & 13.31429 & 26.65159 & 2.26502 & 5.85343 & 11.55903 \\
$(0.5,0.5)$ & 8.05452 & 19.94198 & 39.96466 & 4.37307 & 11.40223 & 22.55128 \\
$(0.75,0.75)$ & 16.02196 & 39.87281 & 78.80769 & 10.18025 & 26.59799 & 50.94848 \\
$(0.9,0.9)$ & 40.01549 & 99.56318 & 193.78700 & 27.03170 & 69.84216 & 129.39040 \\
$(0.25,0.75)$ & 10.69827 & 26.50189 & 53.10912 & 7.38668 & 19.75484 & 41.64418 \\
$(0.1,0.9)$ & 22.09698 & 55.39670 & 108.17150 & 18.96437 & 49.92718 & 92.77766 \\
\hline
\end{tabular}

\begin{tabular}{l|ccc}
\hline \multirow{2}{*}{$\left(p_{1}, p_{2}\right)$} & \multicolumn{3}{|c}{ Logarithmic mean of $M$} \\
\cline { 2 - 4 } & $N=2$ & $N=5$ & $N=10$ \\
\hline$(0.1,0.1)$ & 1.45449 & 2.36539 & 3.06337 \\
$(0.25,0.25)$ & 1.60914 & 2.51012 & 3.20856 \\
$(0.5,0.5)$ & 1.95500 & 2.85091 & 3.54938 \\
$(0.75,0.75)$ & 2.57940 & 3.47319 & 4.15789 \\
$(0.9,0.9)$ & 3.45405 & 4.34048 & 5.01357 \\
$(0.25,0.75)$ & 2.17322 & 3.03959 & 3.70361 \\
$(0.1,0.9)$ & 2.76815 & 3.63691 & 4.29958 \\
\hline
\end{tabular}

\begin{tabular}{l|ccc|ccc}
\hline \multirow{2}{*}{$\left(p_{1}, p_{2}\right)$} & \multicolumn{3}{|c|}{ Skewness of $M$} & \multicolumn{3}{c}{ Kurtosis of $M$} \\
\cline { 2 - 7 } & $N=2$ & $N=5$ & $N=10$ & $N=2$ & $N=5$ & $N=10$ \\
\hline$(0.1,0.1)$ & 1.60896 & 1.77780 & 1.76669 & 7.06338 & 7.94924 & 6.63456 \\
$(0.25,0.25)$ & 1.60117 & 1.72102 & 1.85314 & 6.68347 & 7.01114 & 7.53302 \\
$(0.5,0.5)$ & 1.44783 & 1.57360 & 1.40786 & 5.93274 & 6.53622 & 4.98711 \\
$(0.75,0.75)$ & 1.43156 & 1.45874 & 1.09945 & 6.02005 & 5.86248 & 3.66994 \\
$(0.9,0.9)$ & 1.40786 & 1.24161 & 0.75764 & 6.01493 & 4.48107 & 2.68169 \\
$(0.25,0.75)$ & 2.03801 & 1.87816 & 1.66728 & 9.93744 & 8.15164 & 6.01406 \\
$(0.1,0.9)$ & 1.96784 & 1.92967 & 1.37367 & 8.47793 & 8.12918 & 4.45402 \\
\hline
\end{tabular}

5. Remarks. We have also looked at the above steady-state distributions from alternative angles. Distribution of the number $M$ of transitions comprising a passage time can be evaluated directly through complete enumeration of cases though this is a heavily intensive data-storage process and difficult to carry out for cases where the distribution has a long right tail, that is, when at least one of $p_{1}$ and $p_{2}$ is large.

Once the distribution of $M$ is known, the distribution of passage time $T$ can be obtained as a compound distribution.

ACKNOWLEDGment. The author is indebted to Dr. P. K. Sen, Department of mathematics, Jadavpur University, Calcutta, India, for the kind help and guidance in the preparation of this paper. 


\section{REFERENCES}

[1] J. Brandon and U. Yechiali, A tandem Jackson network with feedback to the first node, Queueing Systems Theory Appl. 9 (1991), no. 4, 337-351.

[2] B. K. Medya, Steady-state behaviour \& simulation studies of a queueing network model, Stochastic Modeling Appl. 4 (2001), no. 1, 59-69.

[3] V. Nguyen, Fluid and diffusion approximations of a two-station mixed queueing network, Math. Oper. Res. 20 (1995), no. 2, 321-354.

[4] S. M. Ross, Applied Probability Models with Optimization Applications, Holden-Day, California, 1970.

[5] G. S. Shedler, Regeneration and Networks of Queues, Applied Probability. A Series of the Applied Probability Trust, Springer-Verlag, New York, 1987.

[6] U. Yechiali, Sequencing an N-stage process with feedback, Probab. Engrg. Inform. Sci. 2 (1988), no. 2, 263-265.

Bidyut K. Medya: Department of Mathematics, Jadavpur University, Calcutta 700032, India E-mail address: medyabk@yahoo.com 


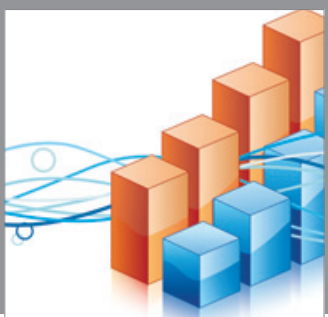

Advances in

Operations Research

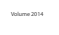

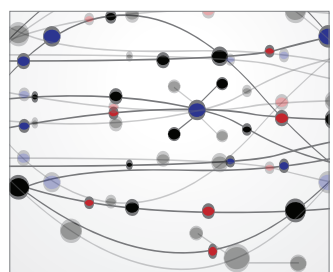

\section{The Scientific} World Journal
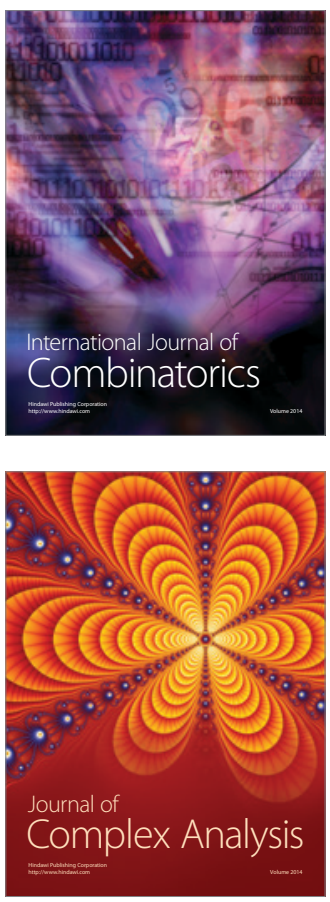

International Journal of

Mathematics and

Mathematical

Sciences
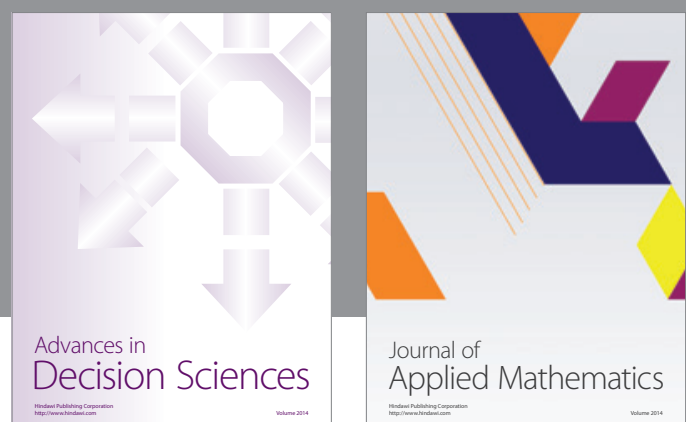

Journal of

Applied Mathematics
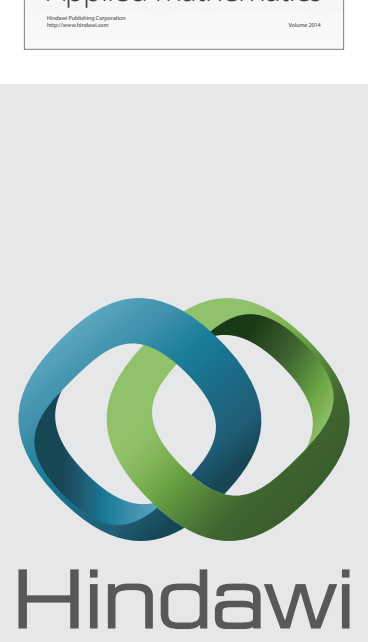

Submit your manuscripts at http://www.hindawi.com
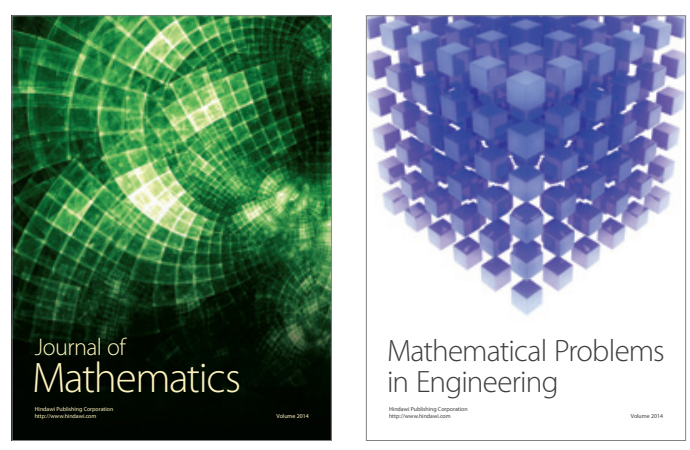

Mathematical Problems in Engineering
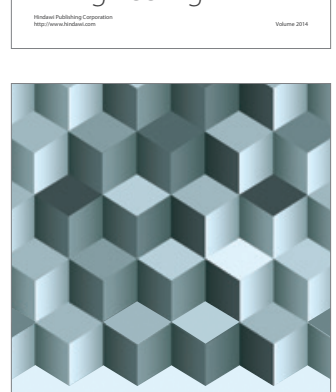

Journal of

Function Spaces
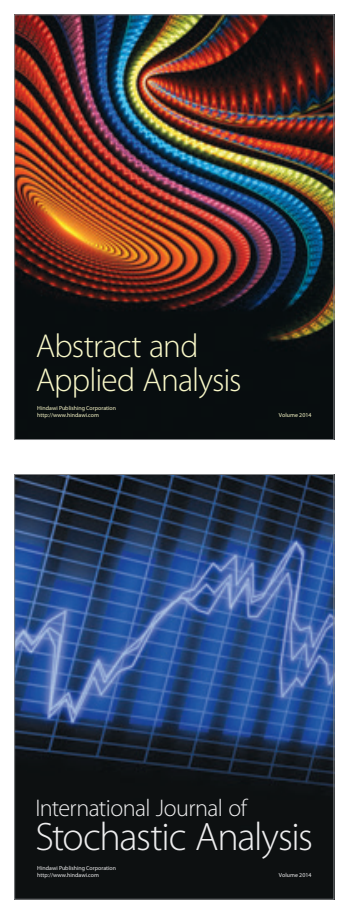

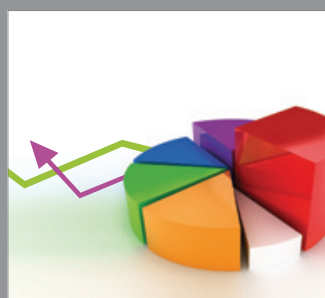

ournal of

Probability and Statistics

Promensencen
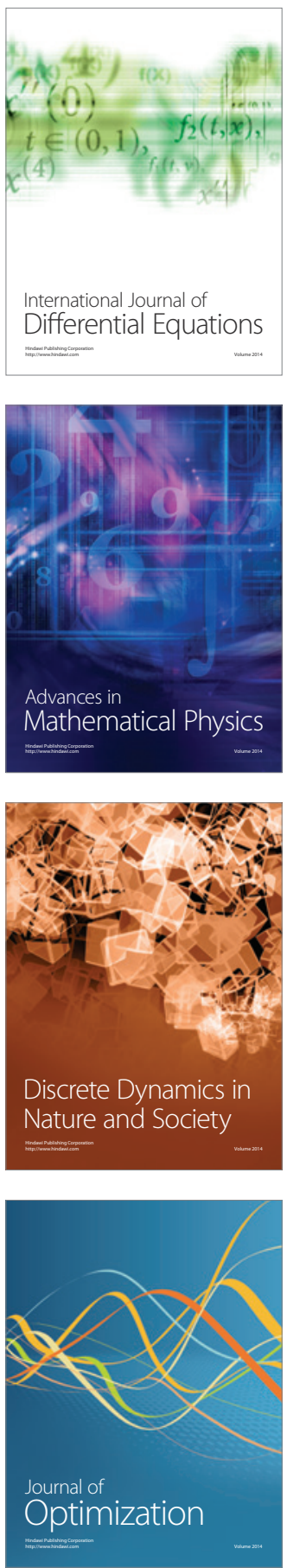